\title{
The Axiom Scheme of Acyclic Comprehension
}

\author{
Zuhair Al-Johar, M. Randall Holmes, and Nathan Bowler
}

\begin{abstract}
A "new" criterion for set existence is presented, namely, that a set $\{x \mid \varphi\}$ should exist if the multigraph whose nodes are variables in $\varphi$ and whose edges are occurrences of atomic formulas in $\varphi$ is acyclic. Formulas with acyclic graphs are stratified in the sense of New Foundations, so consistency of the set theory with weak extensionality and acyclic comprehension follows from the consistency of Jensen's system NFU. It is much less obvious, but turns out to be the case, that this theory is equivalent to $N F U$ : it appears at first blush that it ought to be weaker. This paper verifies that acyclic comprehension and stratified comprehension are equivalent by verifying that each axiom in a finite axiomatization of stratified comprehension follows from acyclic comprehension.
\end{abstract}

The first author, who is a neurologist with an amateur interest in mathematical logic, proposed the criterion of acyclic comprehension for existence of sets (originally under another name) as an approach to the historical paradoxes of set theory and communicated this to the second author. The second author noted that stratified comprehension implies acyclic comprehension, so the scheme of acyclic comprehension is certainly consistent relative to quite weak accepted theories (as the scheme of stratified comprehension is a subtheory of $N F U$, which was shown to be consistent by R. B. Jensen in [6]). The second author also conjectured that the scheme was very weak (meaning not equivalent to full stratified comprehension). The first author realized that one could attack this problem by attempting to prove all propositions in a finite axiomatization of stratified comprehension. (That stratified comprehension is finitely axiomatizable was originally shown by Hailperin in [4], though the axiomatization given there is very unpleasant to work with.) Undaunted by the skepticism of the second author, he proceeded to prove that each of the axioms of the finite axiomatization used in the second author's work [5] (adapted to the Wiener ordered pair of [9]) follows from acyclic comprehension: a précis of his proof can be seen at [1]. That is not the proof given here: here we have given a proof based on the Kura-

Received May 23, 2011; accepted July 12, 2012

2010 Mathematics Subject Classification: Primary 03E70

Keywords: New Foundations, NFU, stratification, acyclic comprehension

(C) 2014 by University of Notre Dame 10.1215/00294527-2377851 
towski pair, though it is actually designed to be as much as possible independent of the implementation of the ordered pair. The third author noticed the importance of certain logical considerations having to do with the effect of definitional expansion on acyclicity, which enabled us to prove a more comprehensive result.

The finite axiomatization used here is nowhere fully verified in the literature, as it is the adaptation to the Kuratowski pair of a finite axiomatization fully verified for the Quine pair in [5], and the change of pair involves some technical work. We include the verification of this finite axiomatization by demonstrating that each of Hailperin's axioms in [4] follow from these axioms.

Definition Let $\varphi$ be a formula in the language of set theory (first-order logic with equality and membership as primitive relations). Let $u$ and $v$ be variables appearing in $\varphi$ (free or bound). We say that a finite sequence $\left\{s_{i}\right\}_{1 \leq i \leq n}$ of variables is a path from $u$ to $v$ in $\varphi$ if and only if $s_{1}=u, s_{n}=v$, and there is an auxiliary sequence $\left\{f_{i}\right\}_{1 \leq i<n}$ of atomic subformulas of $\varphi$ such that for each appropriate index $i$ the atomic subformula $f_{i}$ contains exactly the variables $s_{i}$ and $s_{i+1}$, and for each appropriate index $i, f_{i}$ and $f_{i+1}$ are distinct formulas. The parameter $n$ is called the length of the path.

We say that the formula $\varphi$ is acyclic if and only if it satisfies the following conditions.

(1) There is no more than one path from $u$ to $v$ in $\varphi$ for any variables $u$ and $v$ (including the case where $u$ and $v$ are the same variable, where of course there is a trivial path of length 1 ; for example, the formula $x \in x$ is not acyclic because there is a path of length 2 from $x$ to $x$ in this formula in addition to the trivial path from $x$ to $x$ of length 1).

(2) For any variable $x$ occurring in $\varphi$, either all occurrences of $x$ are free in $\varphi$ or all occurrences of $x$ are bound by the same occurrence of a quantifier.

(3) No atomic formula occurs more than once in $\varphi$.

Note The third author pointed out that one does not want identifications between paths to depend on spurious typographical identifications between variables which are bound in different contexts (or one free and one bound); the second condition did not occur in the original definition.

Definition Let $\varphi$ be a formula in the language of set theory with the property that for each variable $x$, either all occurrences of $x$ are free or all occurrences of $x$ are bound by the same occurrence of a quantifier. We define an undirected multigraph $G_{\varphi}$ as follows: the vertices of this graph are the variables (free or bound) in $\varphi$, and an edge between variables $u$ and $v$ is an occurrence in $\varphi$ of an atomic formula such that the set of variables appearing in the atomic formula is $\{u, v\}$.

Observation A formula $\varphi$ with the property stated under the previous heading is acyclic if and only if $G_{\varphi}$ is acyclic in the usual sense.

Notation for the underlying digraphs Since the formulas we shall be using are long enough to be difficult to digest, we shall include some pictures of the underlying acyclic digraphs to help the reader see the basic structure of the formulas we employ. Pairs of variables in the same atomic formula will be linked by an arrow, such as $x \longleftarrow y$ if the atomic formula is $x \in y$, or $x=y$ if it is $x=y$. Thus, for example, the underlying (cyclic) digraph of the formula expressing extensionality, 
$\forall x . \forall y .(\forall z . z \in x \leftrightarrow z \in y) \rightarrow x=y$, is

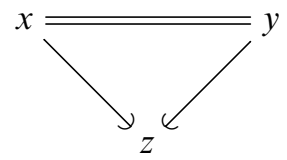

It is important to note that while each variable can appear only once as a vertex in the digraph for a formula, it is permissible for constants (names which have been assigned a fixed reference) to appear more than once, for reasons that will be explained.

Subtleties The definition given above is sensitive to fine details of the way propositional connectives are handled. Here we officially take the view that the primitive propositional connectives are negation and disjunction. ${ }^{1}$ We can use conjunction $p \wedge q$ (defined as $\neg(\neg p \vee \neg q)$ ) and implication $p \rightarrow q$ (defined as $\neg p \vee q$ ) freely, as definitional expansion of these notions does not copy formulas. We must avoid using the biconditional.

Copying of atomic formulas in the expansion of a definition obviously breaks acyclicity. Copying of quantified formulas may break acyclicity in the strict form in which it is defined here, even if we use renaming of bound variables to avoid literal copying of atomic formulas. A formula which occurred in an earlier form of the construction of the converse of a relation was $\left(\forall z . z \in_{2} x \leftrightarrow z \epsilon_{2} y\right)$, where $u \epsilon_{2} v$ is defined as $(\exists w \cdot u \in w \wedge w \in v)$. The formula $\left(\forall z . z \in_{2} x \leftrightarrow z \in_{2} y\right)$ is equivalent to $\left(\forall z .(\exists w . z \in w \wedge w \in x) \leftrightarrow\left(\exists w^{\prime} . z \in w^{\prime} \wedge w^{\prime} \in y\right)\right)$. This in turn expands to $\left(\forall z .\left((\exists w . z \in w \wedge w \in x) \rightarrow\left(\exists w^{\prime} . z \in w^{\prime} \wedge w^{\prime} \in y\right)\right) \wedge\left(\left(\exists w^{\prime \prime} . z \in w^{\prime \prime} \wedge w^{\prime \prime} \in\right.\right.\right.$ $\left.\left.y) \rightarrow\left(\exists w^{\prime \prime \prime} . z \in w^{\prime \prime \prime} \wedge w^{\prime \prime \prime} \in x\right)\right)\right)$, and this is not acyclic.

The underlying digraph is
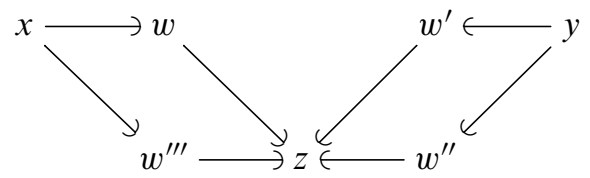

We require that bound variables be diversified as far as possible in acyclic formulas before considering paths, and copying of a quantified formula in which there is a path between free variables with a bound variable on it will cause a failure of acyclicity as here.

However, it is possible to copy a formula freely without impairing acyclicity if it does not contain two distinct free variables connected by a path, by renaming all bound variables in the formula.

Predicates may be introduced by definition (as with $\epsilon_{2}$ above) and used in acyclic formulas if the defining formula of a defined predicate is acyclic, occurrence of variables together in a superficially atomic formula involving a defined predicate is taken to link them for purposes of acyclicity in the same way they would be linked by occurrence together in an atomic formula, and superficially atomic formulas involving defined predicates with more than one argument will not appear more than once (just as an atomic formula cannot). These conditions ensure that definitional expansion of apparently acyclic formulas will in fact produce acyclic formulas when all defined notions are eliminated. 
Axiom scheme of acyclic comprehension For each formula $\varphi$ such that $\varphi$ is acyclic and $A$ does not appear free in $\varphi,(\exists A .(\forall x . x \in A \leftrightarrow \varphi))$ is an axiom.

Observation If $\varphi$ is a formula which is not acyclic, but the formula $\varphi_{x}$ obtained by making each free occurrence in $\varphi$ of a variable other than $x$ a distinct variable is acyclic, then $\left(\exists A .\left(\forall x . x \in A \leftrightarrow \varphi_{x}\right)\right)$ is an instance of acyclic comprehension from which $(\exists A .(\forall x . x \in A \leftrightarrow \varphi))$ follows (since variables free in an instance of comprehension are implicitly universally quantified). This observation allows us to ignore identifications between occurrences of constants in judging whether a formula can be used to define a set, because we can generalize a definition containing a constant by replacing each occurrence of the constant with a different free variable. (By constant we mean here a name which has been assigned a fixed reference; the same remark applies to free variables generally but we use it in practice only for constants in this sense.)

Observation The following axiom is almost always assumed in set theory, as it seems to be part of the underlying concept of what a set is. We add it as an assumption, as it makes the proof more convenient. It is known that stratified comprehension alone interprets stratified comprehension plus weak extensionality, a result of Marcel Crabbé in [2], and we will indicate briefly at the end of the paper how Crabbé's argument can be adapted to acyclic comprehension.

Axiom of weak extensionality We have $(\forall x y z . z \in x \wedge(\forall w . w \in x \leftrightarrow w \in$ $y) \rightarrow x=y$ ): nonempty sets with the same elements are equal.

Definition Acyclic comprehension implies the existence of a set with no elements, as $(\forall y . \neg x=y)$ is an acyclic formula which cannot be true of any $x$. We select one memberless set and let $\emptyset$ represent the selected memberless set. (We may suppose that $\emptyset$ is a new primitive constant with an additional axiom $(\forall x . x \notin \varnothing)$, though this is not strictly necessary.) We then define $\{x \mid \varphi\}$ as the $A$ which witnesses $(\exists A .(\forall x . x \in A \leftrightarrow \varphi))$, if this formula is an instance of acyclic comprehension, and which is equal to $\emptyset$ if it has no elements. This is uniquely determined by weak extensionality and the choice of $\emptyset$.

Observation It is interesting to note that equality can be defined acyclically in terms of membership thus: $x=y$ is equivalent to $(\forall z \cdot x \in z \rightarrow y \in z)$. We do not advocate use of this definition.

Observation Another way to see that certain constants cause no failures of acyclicity if repeated in a formula (namely, ones definable as $\{x \mid \varphi\}$ for an acyclic formula $\varphi$ with no free variables other than $x)$ is to note that $y=\{x \mid \varphi(x)\}$ can be expressed acyclically as $(\forall z . z \in y \rightarrow \varphi(z)) \wedge(\forall w . \varphi(w) \rightarrow w \in y) \wedge$ $((\forall z . \neg(\varphi(z))) \rightarrow y=\emptyset)$ : this can be converted by renaming of bound variables to an acyclic formula as long as there are no free variables in $\varphi(x)$ other than $x$ itself. Then any formula $\psi(\{x \mid \varphi(x)\})$ can be converted to the form $(\exists y . \psi(y) \wedge y=\{x \mid \varphi(x)\})$ (where $y$ is new in the context), which can be made acyclic by renaming bound variables if necessary, if $\psi(y)$ and $\varphi(x)$ are acyclic. Multiple occurrences of the same set abstract can be handled independently with no overlap in variables used.

Definition A formula $\varphi$ in the language of set theory is said to be stratified if and only if there is a function $\sigma$ from variables to integers such that for each atomic 
subformula $x=y$ of $\varphi$ we have $\sigma(x)=\sigma(y)$ and for each atomic subformula $x \in y$ of $\varphi$ we have $\sigma(x)+1=\sigma(y)$.

Observation Any acyclic formula is stratified. For we can choose any variable $x$ in an acyclic formula $\varphi$ and assign it the value 0 under the stratification $\sigma$ to be constructed. This will enable us to determine the value of $\sigma$ at each variable $y$ for which there is a path from $x$ to $y$ : acyclicity ensures that no attempts at multiple assignments of values will be made. If any variables have not been assigned values under $\sigma$, choose one, set the value of $\sigma$ at that variable to 0 , determine values of $\sigma$ at all variables connected to it by paths, and repeat as necessary.

Axiom scheme of stratified comprehension For any stratified formula $\varphi$ in which $A$ is not free, $(\exists A .(\forall x . x \in A \leftrightarrow \varphi))$ is an axiom.

Observations If we assume weak extensionality as well, we can define $\{x \mid \varphi\}$ for each stratified formula $\varphi$ as above, and if $\varphi_{x}$ (defined as above) is stratified, we can show the existence of $\left\{x \mid \varphi_{x}\right\}$ and deduce the existence of $\{x \mid \varphi\}$ as a special case (i.e., we do not need to worry about relative types assigned to free variables in $\{x \mid \varphi\})$.

Main claim The main result of this paper, with whose proof we are occupied from this point on, is that acyclic comprehension and stratified comprehension are equivalent in the presence of weak extensionality. We will indicate briefly after the proof of the main claim how the assumption of weak extensionality could be dispensed with.

Finite axiomatization of stratified comprehension We present a finite list of instances of stratified comprehension which is equivalent to the full scheme. An indication of the proof is found in the second author's [5]. (This is the version modified for use of the Kuratowski ordered pair.) The theorem that stratified comprehension is finitely axiomatizable is due to Hailperin in [4]; details of the implementation (which is due to the second author) are inspired by the reduction of first-order logic to relation algebra in Tarski and Givant [8]. A complete verification that the axioms of Hailperin follow from the axioms given here is found below.

We state the finite axiomatization briefly: complete formal detail of the axioms can be extracted from the verification that they hold in the proof of the main claim. We take weak extensionality as an axiom. We assert the existence of the universal set, which we call $V$, of a complement of each set (only $V$ could have more than one; we specify one complement $\emptyset$ of $V$ to serve in the definition of set abstracts), and of the Boolean union of any pair of sets: sets make up a Boolean algebra. For any set $A$ we assert the existence of the union $\bigcup A$. We assert the existence of singletons $\{a\}$ : from the axioms given so far we can deduce the existence of Kuratowski ordered pairs $(a, b)=\{\{a\},\{a, b\}\}$ for any objects $a, b$.

We assert the existence of Cartesian products. Note that for any set $R$ we can define the relation part of $R$ as $R \cap(V \times V)$. We assert the existence of the converse of any relation (i.e., of the relation part of any set), the relative product of any two relations, the domain of any relation (and so the range since we also have converses of relations), and for any relation $R$ we assert the existence of its singleton image $R^{\iota}$ defined as $\{(\{x\},\{y\}) \mid(x, y) \in R\}$.

We assert the existence of the equality relation, which we denote $[=]$, the projection relations, which take the forms

$$
\pi_{1}=\{((x, y),(x, x)) \mid x, y \in V\}=\{((x, y),\{\{x\}\}) \mid x, y \in V\}
$$


and

$$
\pi_{2}=\{((x, y),(y, y)) \mid x, y \in V\}=\{((x, y),\{\{y\}\}) \mid x, y \in V\}
$$

(these forms are dictated by stratification considerations: the Kuratowski pair is two types higher than its projections), and the inclusion relation $[\subseteq]$.

Proof of the main claim What remains is to show that each of the axioms in the finite axiomatization given is a consequence of acyclic comprehension. We do this with further strictures having to do with making our development as far as possible independent of the choice of implementation of the ordered pair.

Universal set $\quad V=\{x \mid(\exists y \cdot x=y)\}$ exists by acyclic comprehension and is the universal set.

Complement $\{x \mid \neg x \in a\}$ exists by acyclic comprehension and is the complement of $a$. We write this $a^{c}$.

Boolean union $\quad\{x \mid x \in a \vee x \in b\}$ exists by acyclic comprehension and is $a \cup b$; $a \cap b$ can then be constructed as $\left(a^{c} \cup b^{c}\right)^{c}$.

Set union $\quad\{x \mid(\exists y \cdot x \in y \wedge y \in a)\}$ exists by acyclic comprehension and is $\bigcup a$.

Singletons $\quad\{x \mid x=a\}$ exists by acyclic comprehension and is $\{a\}$.

Numeral sets We define 1 as

$$
\left\{y \mid(\exists x . x \in y) \wedge\left(\exists x^{\prime} .\left(\forall z . z \in y \rightarrow z=x^{\prime}\right)\right)\right\}
$$

and note that this exists by acyclic comprehension. We define $2^{*}$ as

$$
\left\{z \mid(\exists x . x \in z) \wedge\left(\exists x^{\prime} y^{\prime} .\left(\forall u . u \in z \rightarrow u=x^{\prime} \vee u=y^{\prime}\right)\right)\right\} .
$$

The underlying digraphs of these formulas are

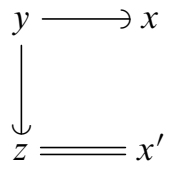

and

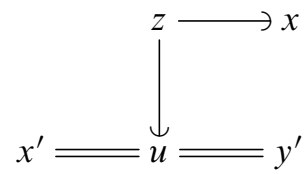

So 1 is the set of singletons (the Frege numeral 1 ), and $2^{*}$ is the set of all unordered pairs (including the singletons, so it is not quite the Frege numeral 2).

It is useful to note that the existence of 1 follows from our finite axiomatization: $1=\operatorname{dom}\left((V \times V)^{\iota}\right)$.

Iterated membership Define $x \in_{1} y$ as $x \in y$. Define $x \epsilon_{k+1} y$ as $(\exists z . x \in z \wedge$ $\left.z \in \in_{k} y\right)$.

Formulas to define projections and pairs We first discuss the pair in the abstract. We suppose that we have acyclic formulas First $(x, p)$ and $\operatorname{Proj}(x, p)$. The intention is that the first formula captures the notion " $p$ is a pair and $x$ is the first projection of the pair $p$," and the second captures the notion " $p$ is a pair and $x$ is one of the projections of the pair $p . "$

Subsequently, we will exhibit specific acyclic formulas implementing the projection and first projection notions for the usual Kuratowski pair (and separately for the Wiener pair), which we will denote by $\operatorname{first}(x, p)$ and $\operatorname{proj}(x, p)$ : the formulas with lowercase names are concrete implementations of the abstract specification of 
First $(x, p)$ and $\operatorname{Proj}(x, p)$. Formulas $\operatorname{Pair}(\mathrm{p})$ ( $p$ is a pair) and $\operatorname{Second}(x, p)$ ( $x$ is the second projection of $p$ ) are defined below in terms of $\operatorname{First}(x, p)$ and $\operatorname{Proj}(x, p)$; once specific formulas $f i r s t(x, p)$ and $\operatorname{proj}(x, p)$ have been introduced implementing First $(x, p)$ and $\operatorname{Proj}(x, p)$ for the Kuratowski (or Wiener) pair, we will have defined implementations pair and second for the Kuratowski (or Wiener) pair of the abstractly specified formulas Pair and Second (the same convention would apply to other notions defined ultimately in terms of First $(x, p)$ and $\operatorname{Proj}(x, p)$, but in most of the argument we leave the choice of a specific implementation of $\operatorname{First}(x, p)$ and $\operatorname{Proj}(x, p)$ open, so notions defined in terms of projections are left abstract, and their names capitalized).

The conditions which must be satisfied for this to be a pair implementation are

$$
(\forall z p .(\operatorname{First}(z, p) \rightarrow \operatorname{Proj}(z, p)) \wedge(\operatorname{Proj}(z, p) \rightarrow(\exists x \cdot \operatorname{First}(x, p))))
$$

(the first projection is a projection, and anything which has a projection [i.e., is a pair] has a first projection),

$$
(\forall x y \cdot(\exists p \cdot \operatorname{First}(x, p) \wedge(\forall z \cdot \operatorname{Proj}(z, p) \leftrightarrow z=x \vee z=y)))
$$

(for any $x$ and $y$ there is an object whose first projection is $x$ and which has $x, y$ as its projections), and

$$
(\forall p \cdot(\forall x y \cdot \operatorname{First}(x, p) \wedge \operatorname{First}(y, p) \rightarrow x=y):
$$

first projections are uniquely determined. We have

$$
(\forall p \cdot(\exists x y \cdot(\forall z \cdot \operatorname{Proj}(z, p) \rightarrow z=x \vee z=y))):
$$

a pair has no more than two projections. This is enough for the second projection to be uniquely determined (and of course the same as the first if the pair has just one projection). We have

$$
\begin{aligned}
& (\forall p q \cdot(\exists x \cdot \operatorname{First}(x, p) \wedge \operatorname{First}(x, q)) \\
& \quad \wedge(\forall y \cdot \operatorname{Proj}(y, p) \leftrightarrow \operatorname{Proj}(y, q)) \rightarrow p=q) .
\end{aligned}
$$

Objects with the same first projection and the same projections are equal, that is, pairs are unique. (It should be noted that this condition is not required for any of our constructions of sets of pairs.) It follows from these conditions that for any $x, y$ there is a unique object $p$ such that $\operatorname{First}(x, p)$ and for each $z, \operatorname{Proj}(z, p)$ if and only if $z=x$ or $z=y$ : we call this object $(x, y)$. It should be clear that though this interface for the pair construction is unusual it does capture precisely the standard notion of an ordered pair.

We can define the notion $\operatorname{Pair}(p)(p$ is an abstract pair) as $(\exists z \cdot \operatorname{Proj}(z, p))$. In our pictorial notation for digraphs, we will use $p-F>x$ and $p-P>x$ to denote the underlying digraphs of $\operatorname{First}(x, p)$ and $\operatorname{Proj}(x, p)$, respectively.

Diagonal We can define the equality relation $[=]$ (the diagonal set) as

$$
\{p \mid \operatorname{Pair}(p) \wedge(\exists x \cdot(\forall z \cdot \operatorname{Proj}(z, p) \rightarrow x=z))\} .
$$

This set exists by acyclic comprehension.

$$
\begin{aligned}
& \text { Abstract definition of the second projection of a pair We define Second }(y, p) \\
& \text { as } \\
& \qquad(\exists P . \operatorname{Proj}(p, P) \wedge(\forall z \cdot \operatorname{Proj}(z, P) \rightarrow \operatorname{Proj}(y, z)) \\
& \quad \wedge(\exists q . q \in[=] \wedge \operatorname{Proj}(q, P)) \wedge \neg(\exists x .(\forall u \cdot \operatorname{Proj}(u, P) \rightarrow \operatorname{First}(x, u)))) .
\end{aligned}
$$


This formula asserts of $p$ and $y$ that there is a pair $P$ such that $P$ has $p$ as one of its projections and $y$ as a projection of each of the projections of $P$ (so $y$ is one of the projections of $p$ ), and $P$ has a projection in [=] (which must be $(y, y)$ ), and the two projections of $P$ do not have the same first projection (so the other projection of $P$ must be of the form $(z, y)$ with $z \neq y)$. Now we see that $y$ is the second projection of both of the projections of $P$ and so of $p$, so this formula implies that $y$ is the second projection of $p$. If $y$ is the second projection of $p$ (and $x$ is the first), then any $P=((z, y),(y, y))$, where $z=x \leftrightarrow x \neq y$, will witness the truth of this formula, so the formula says precisely that $y$ is the second projection of $p$.

Note that the formula is acyclic. The underlying digraph is

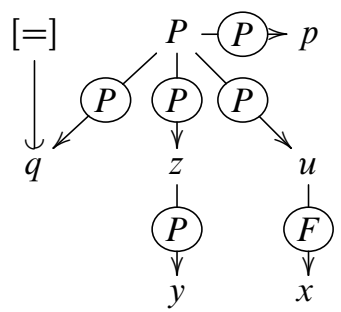

We will use $p-S>y$ to denote the underlying digraph of $\operatorname{Second}(y, p)$.

Observation An implementation of the ordered pair in terms of the first and second projection operators would be greatly to be preferred, but this is not possible. Consider, for example, the ordered pair $(x, y)^{\prime}=(x,\{y\})$, where $(-,-)$ is the usual Kuratowski pair. There are stratified (indeed acyclic) formulas implementing First and Second for this pair, so if there were an acyclic definition of Proj in terms of First and Second, then there would be a stratified implementation of Proj for the pair $(-,-)^{\prime}$, and so the diagonal $\{(x,\{x\}) \mid x \in V\}$, which is the graph of the function $x \mapsto\{x\}$, would be definable as a set in $N F U$, which is well known to be impossible.

Implementation of the Kuratowski pair Suppose that $p$ is the Kuratowski pair $\{\{x\},\{x, y\}\}$. Note that a set is a Kuratowski pair if and only if it is an unordered pair, it has a singleton as an element, all of its elements are unordered pairs, and there is an object which belongs to each of its elements. So we define pair $(p)$ as

$$
\begin{aligned}
& p \in 2^{*} \wedge(\exists q \cdot q \in p \wedge q \in 1) \wedge\left(\forall q^{\prime} \cdot q^{\prime} \in p \rightarrow q^{\prime} \in 2^{*}\right) \\
& \wedge\left(\exists u \cdot\left(\forall q^{\prime \prime} \cdot q^{\prime \prime} \in p \rightarrow u \in q^{\prime \prime}\right)\right) .
\end{aligned}
$$

Note that this is acyclic. The underlying digraph is

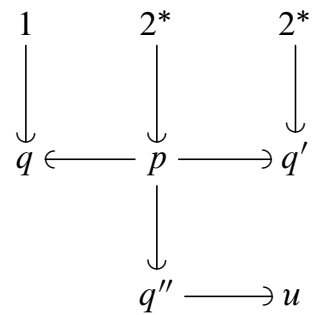


Note further that if $p=\{\{x\},\{x, y\}\}$, then the first projection $x$ of $p$ is the unique object which belongs to all elements of $p$. We define first $(x, p)$ as

$$
\operatorname{pair}(p) \wedge(\forall q \cdot q \in p \rightarrow x \in q) .
$$

Note that this is acyclic.

Note further that the projections of a pair $p=\{\{x\},\{x, y\}\}$ are exactly the elements of the elements of $p$; so we can define $\operatorname{proj}(z, p)$ as

$$
\operatorname{pair}(p) \wedge z \in_{2} p \text {. }
$$

It should be clear that this is an implementation of the ordered pair in the terms given above and that it is essentially the familiar implementation of the ordered pair due to Kuratowski.

An aside: Implementation of the Wiener pair The ordered pair $(x, y)$ was defined by Wiener in [9] as $\{\{\{x\}, \emptyset\},\{\{y\}\}\}$; this is the oldest set-theoretic definition of the ordered pair.

A set $p$ is a Wiener pair if and only if it is an unordered pair, it contains a double singleton, and it contains an unordered pair of a singleton and the empty set; $\operatorname{pair}(p)$ would then be defined as

$$
\begin{aligned}
& p \in 2^{*} \wedge\left(\exists q . q \in p \wedge q \in 2^{*} \wedge \emptyset \in q \wedge(\exists r . r \in q \wedge r \in 1)\right) \\
& \wedge(\exists s . s \in p \wedge s \in 1 \wedge(\exists t . t \in s \wedge t \in 1)) .
\end{aligned}
$$

The underlying (acyclic) digraph is

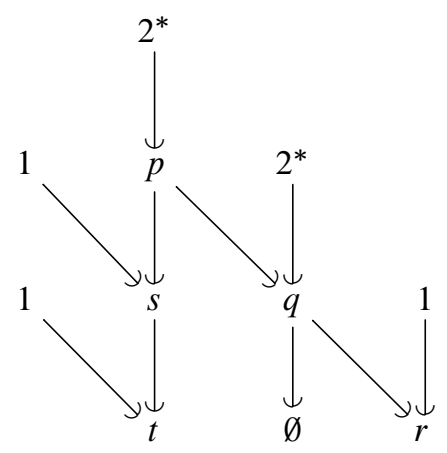

first $(x, p)$ would be definable as

$$
\operatorname{pair}(p) \wedge\left(\exists q . \varnothing \in q \wedge q \in p \wedge x \in_{2} q\right) ;
$$

$\operatorname{proj}(z, p)$ would be definable as

$$
\operatorname{pair}(p) \wedge z \in \in_{3} p .
$$

This completes our interface for the Wiener pair, but it is further worth noting that second $(y, p)$ would admit the very simple definition

$$
\operatorname{pair}(p) \wedge\left(\exists q . q \in 1 \wedge q \in p \wedge y \in_{2} q\right):
$$

the Wiener pair is friendlier to an acyclic treatment if one is willing to use implementation dependent features of the pair. We have a "native" definition of the second projection for the Kuratowski pair, which we have omitted for reasons of space; it is not much if any simpler than the abstract definition of the second projection. 
Note that the verification of the adequacy of our finite axiomatization below depends on the pair being the usual Kuratowski pair, but all other results are indifferent to the choice of pair.

Domains $\quad\{x \mid(\exists p . p \in a \wedge \operatorname{First}(x, p))\}$ exists by acyclic comprehension and is the domain of (the relation part of) $a$.

Cartesian product $\quad a \times b$ can be constructed as $\{p \mid(\exists x \cdot \operatorname{First}(x, p) \wedge x \in a) \wedge$ $(\exists y$.Second $(y, p) \wedge y \in b)\}$. The underlying digraph is

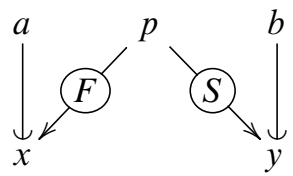

Projections $\quad\{((x, y),(x, x)) \mid x \in V \wedge y \in V\}$, the first projection map $\pi_{1}$, is constructed as

$$
\{p \mid p \in(V \times V) \times[=] \wedge(\exists z \cdot(\forall w \cdot \operatorname{Proj}(w, p) \rightarrow \operatorname{First}(z, w)))\} .
$$

Notice that if the pair is taken to be the Kuratowski pair,

$$
\pi_{1}=\{((x, y),\{\{x\}\}) \mid x \in V \wedge y \in V\} .
$$

$\{((x, y),(y, y)) \mid x \in V \wedge y \in V\}$, the second projection map $\pi_{2}$, is constructed as

$$
\{p \mid p \in(V \times V) \times[=] \wedge(\exists z \cdot(\forall w \cdot \operatorname{Proj}(w, p) \rightarrow \operatorname{Second}(z, w)))\} .
$$

Notice that if the pair is taken to be the Kuratowski pair,

$$
\pi_{2}=\{((x, y),\{\{y\}\}) \mid x \in V \wedge y \in V\} .
$$

A convenient abbreviation We define $\operatorname{FProj}(x, p, F)$ as

$(\exists q r \cdot \operatorname{Pair}(q) \wedge \operatorname{First}(p, q) \wedge \operatorname{Second}(r, q) \wedge \operatorname{Proj}(x, r) \wedge q \in F):$

the point is that $\operatorname{FProj}\left(x, p, \pi_{1}\right)$ is equivalent to $\operatorname{First}(x, p)$ and $\operatorname{FProj}(y$, $\left.p, \pi_{2}\right)$ is equivalent to $\operatorname{Second}(y, p)$. Pictorially, we will represent $\operatorname{FProj}(x$, $p, F)$ by $p-F>x$.

Converses Let $R$ be a set (usually a set relation, i.e., a set of ordered pairs). We can construct

$$
R^{-1}=\{(y, x) \mid(x, y) \in R\}
$$

as

$$
\begin{aligned}
& \left\{p \mid\left(\exists q . q \in R \wedge\left(\forall F . F=\pi_{1} \vee F=\pi_{2} \rightarrow\left(\exists G .\left(F=\pi_{1} \rightarrow G=\pi_{2}\right)\right.\right.\right.\right. \\
& \left.\left.\left.\wedge\left(F=\pi_{2} \rightarrow G=\pi_{1}\right) \wedge(\exists x . F \operatorname{Froj}(x, q, F) \wedge \operatorname{FProj}(x, p, G))\right)\right)\right\} .
\end{aligned}
$$

The underlying (acyclic) digraph is

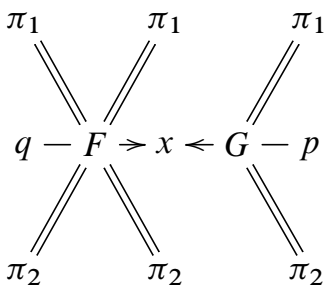


$R^{-1}$ is the converse of the relation part of $R$.

Abstract image-of-a-relation construction We show that for any relation $R$ and acyclic formula $\operatorname{Rel}(a, b)$ we can construct

$$
R^{\operatorname{Re} 1}=\{(u, v) \mid(\exists x y .(x, y) \in R \wedge \operatorname{Rel}(x, u) \wedge \operatorname{Rel}(y, v))\} .
$$

This can be constructed as

$$
\begin{aligned}
\{p \mid & \left(\exists q . q \in R \wedge\left(\forall F . F=\pi_{1} \vee F=\pi_{2}\right.\right. \\
\rightarrow & \left(\exists G .\left(F=\pi_{1} \rightarrow G=\pi_{1}\right) \wedge\left(F=\pi_{2} \rightarrow G=\pi_{2}\right)\right. \\
& \wedge(\exists u v . F \operatorname{Froj}(u, q, F) \wedge \operatorname{FProj}(v, p, G) \wedge \operatorname{Rel}(u, v)))))\} .
\end{aligned}
$$

If we denote $\operatorname{Rel}(u, v)$ by $u-R-v$, the underlying (acyclic) digraph is

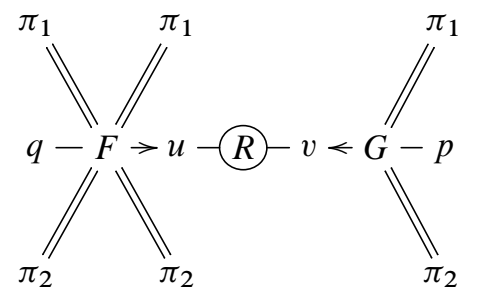

Singleton image (of a relation) We define

$$
R^{\iota}=\{(\{x\},\{y\}) \mid(x, y) \in R\} .
$$

This can be constructed as $R^{\operatorname{Rel} l_{1}}$, where $\operatorname{Rel}_{1}(a, b)$ is taken to be

$$
b \in 1 \wedge a \in b
$$

(i.e., $b=\{a\})$.

Relative products We define a helper set

$$
D=\{((x, z),(y, z)) \mid x, y, z \in V\}:
$$

this can be constructed using the abstract image-of-a-relation construction as $[=]^{\mathrm{Rel}_{2}}$, where $\operatorname{Re}_{2}(a, b)$ is defined as $\operatorname{Second}(a, b)$.

Then $R \mid S$ is constructed as $\left(D \cap\left(R \times S^{-1}\right)\right)^{\operatorname{Re} l_{3}}$, where $\operatorname{Rel}_{3}(p, x)$ is defined as $\operatorname{First}(x, p)$.

Inclusion Construct the set $K$ of all pairs $((x, y),(\{a\},\{a\}))$ such that $a \in x$ as

$$
\begin{aligned}
& \{p \mid p \in(V \times V) \times([=] \cap(1 \times 1)) \\
& \quad \wedge(\exists a \cdot(\forall q r . \operatorname{Proj}(q, p) \wedge \operatorname{First}(r, q) \rightarrow a \in r))\}
\end{aligned}
$$

and the set $L$ of all pairs $((x, y),(\{a\},\{a\}))$ such that $a \in y$ as

$$
\begin{aligned}
& \{p \mid p \in(V \times V) \times([=] \cap(1 \times 1)) \\
& \quad \wedge(\exists a .(\forall q r . \operatorname{Proj}(q, p) \wedge \operatorname{Second}(r, q) \rightarrow a \in r))\} .
\end{aligned}
$$

The existence of the sets $K$ and $L$ follows from acyclic comprehension.

Then $[\subseteq]=\left(\operatorname{dom}\left(K \cap L^{c}\right)\right)^{c} \cap(V \times V)$.

Verification of our finite axiomatization We show that each of the axioms in Hailperin's original finite axiomatization follows from ours and so from acyclic comprehension: 
(P1): $(\forall u v .(\exists \beta .(\forall x .(x \in \beta \leftrightarrow x \in u \downarrow x \in v))))$ : this asserts the existence of $\{x \mid x \in u \downarrow x \in v\}$, where $\downarrow$ is the Sheffer stroke (written $\downarrow$ instead of $\mid$ to avoid confusion with the notation for relative product), that is, $(u \cap v)^{c}$;

(P2): $(\forall \alpha .(\exists \beta .(\forall x y .((\{x\},\{y\}) \in \beta \leftrightarrow(x, y) \in \alpha))))$ : this asserts the existence of singleton images of relations, provided by our axiomatization;

(P3): $(\forall \alpha .(\exists \beta .(\forall x y z .(x, y, z) \in \beta \leftrightarrow(x, y) \in \alpha)))$, where $(x, y, z)$ is defined as $(\{\{x\}\},(y, z))$; define $\alpha^{\iota^{2}}$ as $\left(\alpha^{\iota}\right)^{\iota}$; for each choice of $\alpha$, the corresponding $\beta$ is $\alpha^{\iota^{2}} \mid\left(\pi_{1} \mid \pi_{1}^{-1}\right)$;

(P4): $(\forall \alpha .(\exists \beta .(\forall x y z .(x, z, y) \in \beta \leftrightarrow(x, y) \in \alpha)))$, where $(x, y, z)$ is defined as $(\{\{x\}\},(y, z))$; for each choice of $\alpha$, the corresponding $\beta$ is $\alpha^{\iota^{2}} \mid\left(\pi_{2} \mid \pi_{2}^{-1}\right)$;

(P5): $(\forall \alpha .(\exists \beta .(\forall x y .(y, x) \in \beta \leftrightarrow x \in \alpha)))$ : for each $\alpha$, the corresponding $\beta$ is $V \times \alpha$;

(P6): $(\forall \alpha .(\exists \beta .(\forall x . x \in \beta \leftrightarrow(\forall u .(u,\{x\}) \in \alpha))))$ : the $\beta$ corresponding to a given $\alpha$ is $\bigcup\left(\left(\operatorname{rng}\left(\alpha^{c}\right)\right)^{c} \cap 1\right)$;

(P7): $(\forall \alpha .(\exists \beta .(\forall x y .(y, x) \in \beta \leftrightarrow(x, y) \in \alpha)))$ : this axiom asserts the existence of converses of relations, which is also provided directly by our axioms;

(P8): $(\exists \beta .(\forall x \cdot x \in \beta \leftrightarrow(\exists y \cdot x=\{y\})))$ : this asserts the existence of 1; we remind the reader that $1=\operatorname{dom}\left((V \times V)^{\iota}\right)$;

(P9): $(\exists \beta .(\forall x y .(\{x\}, y) \in \beta \leftrightarrow x \in y))$; a set witnessing this axiom under our axiomatization is $[\subseteq] \cap(1 \times V)$.

Conclusion The proof of the main claim is now complete.

On the dispensability of weak extensionality Marcel Crabbé showed in [2] that the theory $S F$ whose only axiom scheme is stratified comprehension interprets $N F U$, that is, stratified comprehension plus weak extensionality. We give an indication of his approach and how it can be adapted to acyclic comprehension. We obtain a model of $N F U$ from a model of $S F$ as follows: the domains of the two models are the same; the equality relation of the model of $N F U$ is the coextensionality relation of $S F$ (we define $x={ }_{\mathrm{NFU}} y$ as $(\forall z . z \in x \leftrightarrow z \in y)$ ); the membership relation of the model of $N F U$ is the membership relation of $S F$ with its range restricted to those sets which are unions of equivalence classes under coextensionality $\left(x \in_{\mathrm{NFU}} y\right.$ is defined as $\left.x \in y \wedge\left(\forall z w \cdot z==_{\mathrm{NFU}} w \rightarrow(z \in y \leftrightarrow w \in y)\right)\right)$. For details of the proof that this works, see his paper; it is not difficult. What we need to adapt the same result to acyclic comprehension is a verification that the existence of the interpreted equality and membership relations follows from acyclic comprehension: the proof will then go the same way as in [2]. Note that the definition of inclusion in the proof of the main claim is readily modified to give the definition of the converse of inclusion, and the intersection of an inclusion relation and a converse inclusion relation will be a coextensionality relation ("a" rather than "the" because no extensionality is assumed). It must be observed that neither singletons nor Kuratowski pairs are uniquely determined objects in the absence of weak extensionality, but nonetheless, the definition of inclusion relations, and so of coextensionality relations, works correctly. (In fact, none of the constructions of sets of pairs given in the proof of the main claim depends on pairs being unique. Write " $x$ is coextensional with $y$ " as $x \sim y$ : this is equivalent to the acyclic formula $(\exists p \cdot \operatorname{First}(x, p) \wedge \operatorname{Second}(y, p) \wedge p \in$ Coext $)$, where coext is a fixed set implementing the coextensionality relation. We 
can also construct a set relation Inorout implementing the union of inclusion and the disjointness relation on sets (the latter being defined by the formula $\operatorname{Pair}(p) \wedge \neg(\exists x .(\forall y \cdot \operatorname{Proj}(y, p) \rightarrow x \in y)))$ : we write this relation $x$ ? $y$, defined by the acyclic formula $(\exists p \cdot \operatorname{First}(x, p) \wedge \operatorname{Second}(y, p) \wedge p \in$ Inorout $)$. We can then show that there is a set $\Sigma$ of all $y$ which are unions of equivalence classes under coextensionality, namely, $\{y \mid(\forall z .(\exists u .(\forall w . w \in z \rightarrow w \sim u)) \rightarrow z ? y)\}$ : we can then define the membership relation $x \in^{*} y$ of the interpretation as $x \in y \wedge y \in \Sigma$. The outline of the rest of the proof is that it is obvious that the interpreted equality and membership relations satisfy weak extensionality; that they satisfy comprehension follows from the fact that replacing equality and membership with the interpreted equality and membership in an acyclic formula still gives an acyclic formula; and that replacing an object with something coextensional with it in atomic formulas of the interpreted language does not affect truth values, so the same holds for any substitution of coextensional objects in formulas of the interpreted language, so sets witnessing instances of acyclic comprehension expressible in the interpreted language respect coextensionality, which is what is needed for acyclic comprehension to hold in the interpretation.

Final observation The criterion for comprehension presented here is no less a "syntactical trick" than the original stratification criterion of New Foundations (the original system of Quine in [7]) or $N F U$, but it is an interestingly different trick and might possibly find some formal application. A specific suggestion is that acyclic comprehension might have interesting properties from the standpoint of proof theory. We spell out issues related to weak extensionality because in the context of stratified set theory Marcel Crabbé has proved cut elimination for $S F$ (see [3]), but no one has proved cut elimination for $N F U$.

\section{Notes}

1. We note that it is possible to reduce the primitives to the Sheffer stroke (which we write $\downarrow$ to avoid confusion with the notation for relative product), but strictures about copying formulas make this complicated. Define a proposition $t$ as $(\forall x .(\exists y \cdot x=y))$ with the additional remark that in all occurrences of $t$, we rename the bound variables to be different on definitional expansion. Then we can define $\neg p$ as $p \downarrow t$ and $p \vee q$ as $(\neg p) \downarrow(\neg q)$.

2. The use of the biconditional as a primitive was considered by the first author, and the second author considered abandoning restrictions on copying atomic formulas altogether, but the third author convinced us with examples of this sort that extreme care in copying formulas was appropriate in any case, and with some work we were able to complete the proof using the most stringent conditions.

3. We define [ᄃ] here simply as $\{(x, y) \mid(\forall z . z \in x \rightarrow z \in y)\}$, whereas in [5] the urelements are excluded from the domain and range of $[\subseteq]$ : to see that this is harmless it is sufficient to note that we can define the class of urelements as $U=\left(\operatorname{dom}\left([\subseteq]^{c}\right)\right)^{c}-\{\emptyset\}$, and then the restricted subset relation is realized as $[\subseteq] \cap\left(U^{c} \times U^{c}\right)$.

\section{References}

[1] Al-Johar, Z., and M. R. Holmes, "Acyclic comprehension is equal to stratified comprehension," preprint, 2011. 
[2] Crabbé, M., “On NFU," Notre Dame Journal of Formal Logic, vol. 33 (1992), pp. 112-19. Zbl 0766.03031. MR 1149961. DOI 10.1305/ndjfl/1093636013. 14, 22

[3] Crabbé, M., "The Hauptsatz for stratified comprehension: A semantic proof," Mathematical Logic Quarterly, vol. 40 (1994), pp. 481-89. Zbl 0808.03037. MR 1301941. DOI 10.1002/malq.19940400406. 23

[4] Hailperin, T., “A set of axioms for logic," Journal of Symbolic Logic, vol. 9 (1944), pp. 1-19. Zbl 0060.02201. MR 0009753. 11, 12, 15

[5] Holmes, M. R., Elementary Set Theory with a Universal Set, vol. 10 of Cahiers du Centre de logique, Université Catholique de Louvain, Département de Philosophie, Louvainla-Neuve, Belgium, 1998; by permission of the publishers, a version correcting various errata is found at http://math.boisestate.edu/ holmes/holmes/head.ps. Zbl 0959.03001. MR 1759289. 11, 12, 15, 23

[6] Jensen, R. B., "On the consistency of a slight(?) modification of Quine's NF," Synthese, vol. 19 (1968), pp. 250-63.

[7] Quine, W. V., "New Foundations for mathematical logic," American Mathematical Monthly, vol. 44 (1937), pp. 70-80. MR 1523848. DOI 10.2307/2300564. 23

[8] Tarski, A., and S. Givant, A Formalization of Set Theory without Variables, vol. 41 of American Mathematical Society Colloquium Publications, American Mathematical Society, Providence, 1987. Zbl 0654.03036. MR 0920815.

[9] Wiener, N., "A simplification of the logic of relations," pp. 224-27 in From Frege to Gödel: A Sourcebook in Mathematical Logic, 1879-1931, edited by J. van Heijenoort, Harvard University Press, Cambridge, Mass., 1967. Zbl 45.0122.16. 11, 19

\author{
Al-Johar \\ Specialist of Neurology \\ College of Medicine \\ Baghdad University \\ Baghdad \\ Iraq \\ zaljohar@yahoo.com \\ Holmes \\ Department of Mathematics \\ Boise State University \\ 1910 University Drive \\ Boise, Idaho 83725 \\ USA \\ holmes@math.boisestate.edu \\ http://math.boisestate.edu/ holmes \\ Bowler \\ Department of Mathematics \\ Universität Hamburg \\ 20146 Hamburg \\ Germany \\ n.bowler1729@gmail.com
}

